\title{
MULTISYSTEMIC INFLAMMATORY SYNDROME: A CASE SERIES
}

\author{
Jean Paulo Veronese de Souza1, , Vanessa de Quadros Martins, Karol Levien Dora², Fernanda de Oliveira Chiaradia², Marilia \\ Comissoli Brust² ${ }^{2}$, Deise Marcela Piovesan', Bárbara Mendes da Silva' ${ }^{1}$, Marcelo Maltchik', Roberta Casanova Wilhelms', Camila \\ Schafer ${ }^{1}$, Markus Bredemeier', lloite Maria Scheibel ${ }^{2}$
}

1. Hospital Nossa Senhora da Conceição, Porto Alegre (RS), Brazil. 2. Hospital da Criança Conceição, Porto Alegre (RS), Brazil.

*Corresponding author: jeanpvs713@gmail.com

\section{BACKGROUND}

Multisystemic inflammatory syndrome (MIS) is a rare complication of infection by the SARS-CoV-2 virus, with an estimated incidence of 2 cases per 100,000 children. The syndrome manifestations resemble those of Kawasaki's disease (KD), but serious complications, such as shock and myocardial dysfunction, are more frequent.

\section{MATERIALS AND METHODS}

We report here a series of four cases of children with MIS, whose hospitalization occurred between March and September 2020. Medical records of patients were reviewed; all patients were tested for SARS-CoV-2 infections by serology (IgG and IgM). Case series: one patient presented incomplete and three the complete form of KD. The age of the patients ranged from 6 months to 11 years, three of whom were male. All of them presented fever for more than 5 days, nonpurulent conjunctivitis, rash, changes in the oral mucosa, mainly fissures or perioral lesions, and only one presented extremity changes. All patients had gastrointestinal symptoms, abdominal pain or diarrhea. Elevation of C-reactive protein and ferritin occurred in all children, with elevation of erythrocyte sedimentation rate in only two. Hematimetric manifestations consisted of a drop in hemoglobin, leukocytosis with leftsided deviation and thrombocytosis in three patients, and lymphopenia and thrombocytopenia in one. Only one of the patients did not show elevated transaminases. Of the patients in our case series, there were two children with coronary complications, one with isolated coronary artery aneurysm and other with giant left coronary and its branches aneurysms. Only two presented positive serological tests for SARS-CoV-2 virus (both these cases did not present coronary artery aneurisms). The two patients with coronary aneurysm did not respond to two doses of human IGIV, but did responded quickly to endovenous corticosteroids; the other two patients responded to oral corticosteroids. The patients recovered from their illness and are now being followed-up on an outpatient basis. All patients received maintenance therapy with aspirin, but one of them (with partial improvement in coronary aneurisms) also maintains treatment with warfarin and carvedilol.

\section{CONCLUSION}

We report here 4 cases of children with MIS, a new clinical entity in the context of a SARS-CoV-2 virus pandemic. The meaning and long-term complications of this condition are still unknown; regular clinical follow-up of patients and scientific technical collaboration between centers is necessary to define prognosis and the response to therapy in the context of MIS. 